\title{
Le droit suisse des voyages à forfait à la lumière de quelques plateformes de réservation en ligne
}

\begin{abstract}
Alison Notaro *
Avec l'avènement du commerce en ligne sont apparues de nombreuses plateformes de réservation de voyages, à l'image de booking.com ou d'ebookers.ch, lesquelles sont venues bouleverser les codes traditionnels du secteur du tourisme. La présente contribution se propose d'analyser le fonctionnement de ces nouveaux acteurs à la lumière du droit suisse des voyages à forfait, avec quelques incursions du droit européen. L'on constatera ainsi qu'il n'est pas si simple de rattacher ces plateformes à notre législation, laquelle tarde, comme souvent en droit de la consommation, à s'adapter aux évolutions économiques et sociales.
\end{abstract}

I. Introduction. 42

II. Le fonctionnement de différentes plateformes de réservation en ligne : un bref panorama 42

1. Booking.com : une prestation seulement....................................................42

2. Ebookers.ch : les combinaisons .................................................................43

3. Hotelplan.ch : l'agence virtuelle..........................................................44

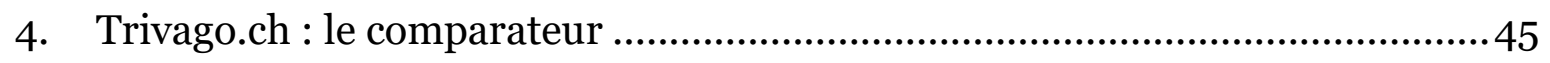

III. Les plateformes de réservation sous la loupe du droit suisse ............................45

1. La question de l'intégration des conditions générales..................................45

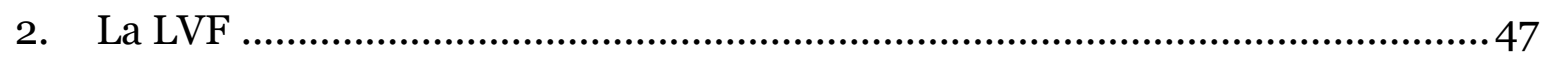

3. L'applicabilité de la LVF aux plateformes de réservation en ligne..................51

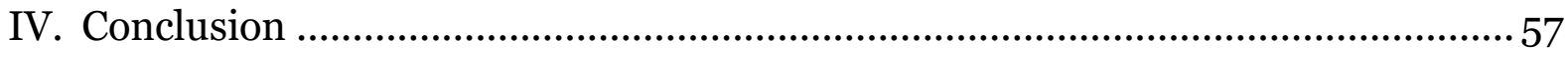

Zitiervorschlag: Alison Notaro, Le droit suisse des voyages à forfait à la lumière de quelques plateformes de réservation en ligne, in: sui-generis 2018, S. 41

URL: $\quad$ sui-generis.ch/58

DOI: $\quad$ https://doi.org/10.21257/sg.58

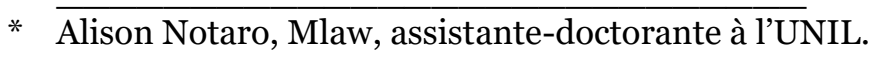

Dieses Werk ist lizenziert unter einer Creative Commons Namensnennung - Weitergabe unter gleichen Bedingungen 4.0 International Lizenz. 


\section{Introduction}

1 Que l'on cherche un hôtel bien placé en Côte d'Azur, un vol pour New York ou un safari en Tanzanie, Internet est pour beaucoup un outil de prédilection. Or, qui dit Internet dit également une diversité d'offres toutes plus alléchantes les unes que les autres, mais aussi des risques pour le consommateur, lequel, sous le coup de l'excitation quant à des vacances bien méritées, oublie parfois le nuage juridique qui plane sur lui.

2 Depuis plusieurs années, des plateformes de réservation en ligne sont arrivées sur la toile afin de grignoter une part d'un gâteau toujours plus grand qu'est le secteur du tourisme. Toutefois, elles ne proposent pas toutes le même type de prestation, raison pour laquelle nous allons d'abord procéder à une typologie de quelques plateformes choisies (II).

La Suisse s'est dotée d'un outil spécifique en matière de tourisme, la Loi fédérale sur les voyages à forfait (ci-après LVF) ${ }^{1}$. Comme tout instrument juridique, il convient d'en déterminer le champ d'application, tant sous l'angle matériel que personnel; nous tenterons ainsi d'examiner si cette réglementation s'applique aux différentes plateformes de réservation (III). Si tel n'est pas le cas, il sera intéressant d'analyser quels autres instruments offre le droit suisse à ce nouveau mode de consommation. Nous traiterons également du droit européen et plus brièvement de problématiques liées au droit international privé.

\section{Le fonctionnement de différentes plateformes de réservation en ligne : un bref panorama}

4 Lorsque le consommateur effectue une recherche sur Internet, apparaitront à l'écran de nombreuses plateformes, lesquelles ne fonctionnent pas de la même manière notamment pour des raisons de marketing et de concurrence. Or, pour appréhender juridiquement ces plateformes, il convient dans un premier temps d'expliquer leurs méthodes commerciales puis de détacher, souvent à l'aide de leurs conditions générales (ciaprès : CG), quelques prémices légales qui nous aideront à les rattacher au droit suisse, ce que nous examinerons au chapitre suivant.

5 Plusieurs modèles se démarquant en pratique, notre choix s'est porté sur quatre exemples. L'on mentionnera ici que certaines compagnies aériennes, à l'image d'EasyJet, se sont également lancées dans le business des voyages à forfait en proposant des «séjours ${ }^{2}$; toutefois, contrairement aux plateformes que nous allons analyser, la compagnie aérienne opère en principe elle-même le transport, soit l'une des prestations caractéristiques du voyage à forfait.

\section{Booking.com : une prestation seulement}

6 En se rendant sur le site Internet de Booking, il suffit d'entrer dans le champ de recherche une destination avec une

\footnotetext{
D’après les conditions de réservation d'EasyJet, il s'agit de voyages à forfait, soumis au droit suisse lorsque le voyageur opère la réservation depuis le site helvétique de la compagnie.
} 
plage temporelle 3 et la plateforme trouve une pléthore de résultats : il ne reste plus qu'à choisir un hôtel parmi les propositions, puis finaliser la réservation en indiquant ses coordonnées personnelles et évidemment en passant à la caisse ; rien de bien sorcier donc !

7 Booking.com propose notamment les prestations suivantes :

- L'hébergement, soit la réservation d'une chambre d'hôtel, d'une chambre dans une auberge ou la location d'un appartement ${ }^{4}$;

- Le vol : soit plusieurs choix, avec des compagnies différentes et des sites de réservation différents ;

- La location de voiture: le fonctionnement est le même que pour les vols ;

- La réservation de restaurant: booking.com nous redirige alors vers un autre site, opentable.com, afin d'effectuer la réservation.

La question se pose de savoir comment booking.com se situe-t-elle vis-à-vis de ses clients ? D'après les CG d'utilisation de Booking, "(...) nous [(Booking.com B.V. et ses partenaires (de distribution) affiliés)] fournissons un portail en ligne

Par exemple, le client inscrit «Lisbonne » avec comme plage temporelle du 23 décembre au 30 décembre 2018.

4 Ce qui n'est pas sans rappeler une autre plateforme en ligne, Airbnb, laquelle pose un certain nombre de questions, voir à ce propos RiemerKafka Gabriela/Studer Viviana, Digitalisierung und Sozialversicherung - einige Gedanken zum Umgang mit neuen Technologien in der Arbeitswelt, in : G. Riemer-Kafka et al., SZS 2017 p. 354 . grâce auquel les Fournisseurs de Voyage peuvent proposer leurs produits et services à la commande, à l'achat, à la réservation ou à l'emploi, dans le cadre de leur étendue commerciale (par ex., B2B ou $B 2 C$ ), et grâce auquel les visiteurs de la Plateforme peuvent consulter, rechercher ou comparer les produits et services concernés et passer une commande, réserver ou encore effectuer un achat ou un paiement (pour le Service de Voyage choisi). En utilisant le Service de Voyage (par ex., en effectuant une Réservation de Voyage par l'intermédiaire du Service de Voyage), vous vous engagez dans une relation contractuelle directe avec le Fournisseur de Voyage auprès duquel vous avez effectué une réservation ou acheté un produit ou service (le cas échéant). À partir du moment où vous effectuez une Réservation de Voyage, nous agissons uniquement en qualité d'intermédiaire entre vous et le Fournisseur de Voyage, en transmettant les détails de votre réservation au $(x)$ Fournisseur(s) de Voyage concerné(s) et en vous envoyant un e-mail de confirmation pour et au nom du Fournisseur de Voyage. Booking.com ne (re)vend, loue ou propose aucun produit ou service (de voyage)».

9 Ainsi, booking.com se considère-t-elle comme un simple intermédiaire entre le "fournisseur », soit par exemple l'hôtel $\mathrm{X}$, et le « visiteur de la plateforme », soit le client.

\section{Ebookers.ch : les combinaisons}

10 La plateforme ressemble sensiblement à ses concurrentes; il suffit de choisir une destination ainsi qu'un espace temporel, puis préciser si l'on désire uniquement un vol ou un hôtel ou si l'on souhaite une 
combinaison des deux. Ensuite, plusieurs choix s'offrent au voyageur qui sélectionnera en quelques clics de souris ce qui lui correspond le mieux.

11 Comment considérer cette plateforme? D'après les CG de réservation d'ebookers.ch, il sied de distinguer deux types d'offres :

- Le vol OU l'hôtel : selon les CG, il s'agit de services de transport aérien et de services d'hébergement. Il est précisé que ceux-ci sont offerts séparément et soumis aux règles et restrictions du fournisseur.

Il n'est toutefois pas indiqué précisément quel est le rôle d'ebookers.ch dans la fourniture de ces services ; nous tenterons de décrypter juridiquement quel(s) type(s) de contrat(s) se cachent derrière cette constellation.

- Le vol ET l'hôtel (le « séjour combiné ») : les CG d'ebookers.ch sont très claires à ce propos: les services de transport aérien et d'hébergement peuvent être offerts comme une partie d'un voyage à forfait, lequel est défini au chiffre 3.4 comme " (...) un voyage, à un prix forfaitaire, pour un séjour de plus de 24 heures (ou incluant une nuitée), dans lequel au moins deux des trois éléments suivants sont offerts : transport - hébergement - service touristique qui ne fait pas partie du transport ou de l'hébergement, mais constitue une partie importante du prix total (...). La vente de voyages à forfait est régie par la loi fédérale sur les voyages à forfait du 18 juin 1993 ».
12 Dans cette configuration et contrairement à booking.com, ebookers.ch ne se place pas en simple qualité d'intermédiaire, mais organise un véritable voyage à forfait, comme le ferait une agence de voyage classique ayant pignon sur rue.

\section{Hotelplan.ch : l'agence virtuelle}

13 Hotelplan.ch est la plateforme d'une véritable agence de voyages au sens physique du terme, comme il en existe encore en Suisse. Elle propose différentes prestations, comme la combinaison vol et hôtel (mais il est possible de réserver le vol ou l'hôtel séparément), les croisières, les circuits ainsi que la location de voitures. A première vue, rien ne semble bien différent de booking.com; or, à la lecture des CG de contrat et de voyage de $\mathrm{MTCH}$ SA5, l'on constate que "MTCH organise des voyages à votre intention. Nous nous engageons à (...) organiser votre voyage du début à la fin selon les données et descriptions figurant dans les prospectus et les autres publications de MTCH, (...) mettre à votre disposition l'hébergement convenu, (...) vous fournir toutes les autres prestations comprises dans le forfait que vous aurez choisi ».

14 Ainsi, hotelplan.ch ne se positionne pas en simple intermédiaire, lequel a pour fonction essentielle de mettre en relation les clients et fournisseurs de prestations de voyage, mais organise un voyage (à forfait ?) de $\mathrm{A}$ à $\mathrm{Z}$ en faisant éventuellement appel à des fournisseurs.

5 Soit Hotelplan Suisse, la société détentrice de la marque Hotelplan. 


\section{Trivago.ch : le comparateur}

15 Alors que les plateformes examinées précédemment proposaient plusieurs services de voyage, trivago.ch focalise son activité sur l'hôtellerie. Son fonctionnement est très simple : elle [[Trivago ?]] se base sur les indications que le client insère dans la barre de recherche, puis propose une multitude de choix, dans un esprit de comparaison (ou de concurrence), puisque les hôtels apparaissent à différents prix. Par exemple, l'hôtel X est indiqué à $\mathrm{CHF} 250$ sur son propre site Internet, à CHF 300 sur expedia.fr et à CHF 350 sur booking.com ${ }^{6}$.

Cela signifierait-il que trivago.ch se cantonne au simple rôle de vitrine du net, permettant de voir en un clin d'œil plusieurs offres? La lecture des "AGB » (Allgemeine Geschäftsbedingungen)7 de ladite plateforme ne laisse planer aucun doute : trivago.ch n'apparaît pas en tant que voyagiste ou partenaire contractuel de la réservation, mais fournit simplement le lien technique pour accéder à la page de réservation de l'hôtel correspondante ${ }^{8}$. Il est en outre précisé que trivago.ch n'entre pas en relation contractuelle avec l'utilisateur de la plateforme, soit le client, pas plus qu'elle ne l'est avec les hôteliers.

\section{Les plateformes de réservation sous la loupe du droit suisse}

17 Il s'agit ici d'examiner dans un premier temps la question des CG utilisées par les plateformes et leur intégration (1). Puis, nous analyserons l'applicabilité matérielle et personnelle de la LVF aux dites plateformes (2 et 3 ).

\section{La question de l'intégration des conditions générales}

18 Nous avons vu dans la typologie des différentes plateformes que celles-ci utilisent toutes des CG sous des dénominations variées telles que «conditions générales d'utilisation », « conditions générales de réservation » ou encore « $\mathrm{AGB} »$.

19 D'après une définition bien ancrée en droit suisse9, les CG sont "des clauses contractuelles préformulées qui décrivent de manière générale tout ou partie du contenu d'éventuels contrats». Les CG doivent toutefois être intégrées au contrat des parties pour avoir un effet juridique propre ${ }^{10}$, ce qui signifie que les parties doivent s'accorder sur le fait que les CG interviendront en sus de leur ac-
6 Il est intéressant de constater que la concurrence fait rage entre les plateformes et qu'elles n'hésitent pas à se comparer !

7 L'on précisera ici que les CG de trivago.ch sont à la fois en anglais et en allemand, ce qui ne facilite pas la tâche du consommateur.

8 Sous c. 2.2 des AGB de trivago.ch_: «trivago does not appear as a tour operator or contract partner of the booking, but merely provides the technical link to the respective hotel booking page ».
9 Arrêt du Tribunal fédéral 4P.135/2002 du 28 novembre 2002, Kuonen Nicolas, Le contrôle des conditions générales : l'envol manqué du phénix, SJ 2014 II p.4, Pichonnaz Pascal/Fornage AnneChristine, Le projet de révision de l'art. 8 LCD Une solution appropriée à la difficulté de négocier des conditions générales, RSJ 106/2010 p. 288.

10 ATF 118 II 295 = JdT 1993 I 400, P. Pichonnaz, in : V. Martenet/ P. Pichonnaz (édit.), Commentaire romand, Loi contre la concurrence déloyale, Bâle 2017, $\mathrm{n}^{\circ} 44$ ad art. 8 LCD, Fornage Anne-Christine, Le contrat d'assurance protection juridique: quelques clauses choisies, in : B.

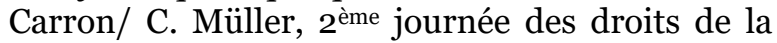
consommation et de la distribution, Bâle 2016, p. $5 \mathrm{n}^{\circ} 6$. 
cord principal et régiront leurs relations contractuelles ${ }^{11}$.

La question de l'intégration des CG pose quelques difficultés en matière de contrats conclus par voie électronique. Nous rejoignons Pichonnaz lequel estime que dans cette situation, le destinataire doit avoir accès aux CG par le biais d'un téléchargement, d'une sauvegarde (par ex. en format PDF) ou d'une impression ${ }^{12}$. Certains auteurs précisent encore que "les conditions générales doivent être présentées avant la conclusion du contrat, soit avant que le bouton pour la confirmation de la commande n'ait été sélectionné »13. Aussi, nous sommes d'avis qu'il y a intégration lorsque le client se contente de cocher une case "lu et approuvé » et qu'un lien lui permet d'accéder directement au contenu des CG ; l'on devrait parler ici d'intégration globale, car le voyageur adhère aux CG sans les remettre en question, voire même sans prendre la peine de les examiner ou de les lire ${ }^{14}$.

L'on précisera en outre que l'art. 4 LVF prévoit que l'organisateur ou le détaillant doit communiquer par écrit au consommateur toutes les clauses du contrat avant sa conclusion; les clauses du con-

11 Pichonnaz (n. 10), ${ }^{\circ} 44$ ad art. 8, Fornage (n. 10), p. $5 \mathrm{n}^{\circ} 6$, Tercier Pierre/Pichonnaz Pascal, Le droit des obligations, 5ème éd., Genève et al. 2012, p. 193 n869.

12 Pichonnaz (n. 10), $\mathrm{n}^{\circ} 53$ ad art. 8, Fornage (n. 10), p. $6 \mathrm{n}^{\circ} 7$, Schwenzer Ingeborg, Schweizerisches Obligationenrecht, Allgemeiner Teil, 6ème éd., Berne 2012, $n^{\circ} 45.06 a$.

13 Kramer Ernst/Probst Thomas/Perrig Roman, Schweizerisches Recht der Allgemeinen Geschäfstbedingungen, Berne 2016, $\mathrm{n}^{\circ} 161$, Pichonnaz (n. 10), $\mathrm{n}^{\circ} 53$ ad art. 8.

14 ATF 126 III $278=$ JdT 2000 I 579, arrêt du Tribunal fédéral 5 C.74/2002 du 7 mai 2002, Fornage (n. 10), p. $7 \mathrm{n}^{\circ} 10$. trat peuvent toutefois être transmises au consommateur sous une autre forme appropriée mais dans ce cas elles doivent lui être confirmées, avant la conclusion du contrat, par une copie écrite. Selon Stauder, bien que le consommateur ait accès aux CG en ligne et puisse les lire sur l'écran, voir les stocker dans ses fichiers, " une confirmation par une copie écrite sur support papier reste nécessaire (...) il ne suffit pas que le consommateur puisse obtenir une copie des $C G V$ [conditions générales de vente, ndlr] au moyen d'une imprimante »15. Nous ne sommes pas de cet avis ${ }^{16}$ et ce notamment au regard du nouveau droit européen $^{17}$ lequel prévoit que la copie ou la confirmation du contrat de voyage à forfait doit être donnée sur un support durable, lequel se définit comme " tout instrument permettant (...) de stocker des informations (...) d'une manière permettant de s'y reporter ultérieurement pendant un laps de temps adapté aux fins auxquelles les informations sont destinées et qui permet la reproduction à

15 B. Stauder, in : L. Thévenoz/F. Werro (édit.), Commentaire romand, Code des obligations I: art. 1 - 529, Loi sur le crédit à la consommation, Loi sur les voyages à forfait, Bâle et al. 2004, $\mathrm{n}^{\circ} 8$ ad art. 4 LVF. Plus nuancé : Wiede Andreas, Reiserecht, Schweizer Handbuch zu den Verträgen über Reiseleistungen, Zurich et al. 2014, p. $240 \S 812$.

16 Voir ég. V. Roberto, in : H. Honsell et al. (édit.), Basler Kommentar, Obligationenrecht I : art. 1529 OR, 6ème éd., Bâle 2015, ${ }^{\circ} 4$ ad. art. 4 LVF, lequel estime que la règlementation de l'art. 4 LVF est dépassée et qu'une notification électronique est efficace.

17 Cf. art. 7 de la Directive 2015/2302/UE du Parlement européen et du Conseil du 25 novembre 2015 relative aux voyages à forfait et aux prestations de voyage liées, modifiant le règlement $(\mathrm{CE})$ $\mathrm{n}^{\circ}$ 2006/2004 et la directive 2011/83/UE du Parlement européen et du Conseil et abrogeant la directive 90/314/CEE du Conseil, JO L 326/1 du 11 décembre 2015 (ci-après Directive 2015/2302/UE). 
l'identique des informations stockées $»^{18}$. Le voyageur est toutefois en droit de demander un exemplaire papier si le contrat à forfait a été conclu en la présence physique et simultanée des parties. De plus, si le contrat est un contrat hors établissement au sens de l'art. 2 point 8 de la Directive 2011/83/UE, un exemplaire ou la confirmation du contrat de voyage est fournie au voyageur sur support papier ou, moyennant son accord, sur un support durable.

Ces réflexions trouvent ancrage dans la «parentalité » entre le droit suisse des voyages à forfait et le droit européen. En effet, dans les années nonante et suite au refus du peuple suisse d'adhérer à l'Espace économique européen ${ }^{19}$, le législateur suisse s'est doté de la LVF dans le cadre du processus Swisslex afin notamment d'assurer la protection des consommateurs ${ }^{20}$. La LVF était alors basée sur l'ancienne Directive 90/314/CEE ${ }^{21}$, laquelle a été abrogée par la nouvelle Directive 2015/2302/UE. Malgré ces changements, la LVF n'a pas été modifiée ; les questions relatives à l'adaptation législative et à l'interprétation du droit suisse à la lumière du droit européen ayant été

18 Art. 3 § 11 de la Directive 2015/2302/UE.

19 Message du 24 février 1993 sur le programme consécutif au rejet de l'Accord EEE (FF 1993 I 761).

20 Stauder (n. 15), $\mathrm{n}^{\circ} 2$ des remarques préliminaires à la LVF, Marchand Sylvain, De l'helvéticocompatibilité de la Loi fédérale du 18 juin 1993 sur les voyages à forfait, PJA 1994 p. 721 . Pour d'autres objectifs cf. Fornage Anne-Christine, $L a$ mise en œuvre des droits du consommateur contractant, Etude de droit suisse avec des incursions en droit de l'Union européenne, en droit anglais, français et allemand, thèse de doctorat, Berne 2011, p. $143 \S 625$.

21 Directive 90/314/CEE du Conseil du 13 juin 1990 concernant les voyages, vacances et circuits à forfait, JOCE L158 du 23 juin 1990. largement traitées tant par la doctrine ${ }^{22}$ que par la jurisprudence ${ }^{23}$, nous nous permettons d'y renvoyer le lecteur.

\section{La LVF}

23 Vieille de plus de 20 ans, la LVF n'a pas subi de modifications depuis son adoption et n'a pas fait les gros titres de la jurisprudence fédérale, hormis quelques arrêts. Efficace donc cette loi ? Nous nous proposons de répondre à cette question en analysant le champ d'application de la LVF par rapport aux plateformes examinées précédemment.

\section{a) Le champ d'application de la loi}

24 L'art. 1 de la LVF définit le voyage à forfait comme la combinaison fixée préalablement d'au moins deux des prestations suivantes, soit le transport, l'hébergement ou les autres services touristiques non accessoires au transport ou à l'hébergement représentant une part importante dans le forfait, lorsque cette combinaison est offerte à un prix global et qu'elle dépasse vingt-quatre heures ou inclut une nuitée.

25 Comme le relève Stauder ${ }^{24}$, plusieurs points ressortent de cette définition :

- La combinaison d'au moins deux prestations touristiques principales :

22 Wiede (n. 15), pp. 152s., Fornage (n. 20), pp. 167s., Roberto (n. 16), $\mathrm{n}^{\circ}$ 1a des remarques préliminaires à la LVF.

23 ATF 129 III 335, c. 6: «Die Angleichung in der Rechtsanwendung darf sich dabei nicht bloss an der europäischen Rechtslage orientieren, die im Zeitpunkt der Anpassung des Binnenrechts durch den Gesetzgeber galt. Vielmehr hat sie auch die Weiterentwicklung des Rechts, mit dem eine Harmonisierung angestrebt wurde, im Auge zu behalten.».

24 Stauder (n. 15), $\mathrm{n}^{\circ} 2$ ad art. 1 LVF. 
Le voyage à forfait s'inscrit dans le cadre du tourisme. Il est ainsi nécessaire, pour que la LVF s'applique, que le contrat ait pour socle des «prestations touristiques », qui peuvent être le transport, l'hébergement, voir même d'autres prestations comme des visites guidées ou des spectacles ${ }^{25}$. A titre d'exemple, le Tribunal fédéral a considéré ${ }^{26}$ que la simple mise à disposition d'un bateau de plaisance n'entre pas dans le champ d'application de la LVF, alors que tel est le cas de la croisière qui combine effectivement plusieurs prestations touristiques (transport, hébergement, visites, spectacle etc.).

Enfin, il faut souligner que ces différentes prestations doivent être combinées : la proposition d'une seule d'entre elle, par exemple la location d'un appartement de vacances, ne constitue pas un voyage à forfait 27 .

- Cette combinaison doit être préalable :

La LVF exige encore que la combinaison des prestations touristiques soit préalable, en ce sens que l'organisateur du voyage doit le préparer en amont, comme un produit fabriqué, puis le proposer au public; tel est le cas de l'agence de voyages qui propose des voyages tout compris $^{28}$. Or, cette condition peut manquer si l'on songe aux évolutions récentes dans le secteur du tourisme.

25 Tercier Pierre/Bieri Laurent/Carron Blaise, Les contrats spéciaux, 5ème édition, Genève et al., p. $868 \S 75 \mathrm{n}^{\circ} 5859$, Stauder (n. 15$), \mathrm{n}^{\circ} 5$ ad art. 1 LVF, Roberto (n. 16), $n^{\circ} 5$ ad art. 1 LVF.

26 ATF 139 III 217.

27 Stauder (n. 15), ${ }^{\circ} 6$ ad art. 1 LVF.

28 Stauder (n. 15), $\mathrm{n}^{\circ} 8$ ad art. 1 LVF.
29 Prenons par exemple ebookers.ch, dont on a vu qu'elle propose des vols et chambres d'hôtel en fonction de critères préétablis par le voyageur. Dans cette situation, le client choisit et compose en quelque sorte lui-même son voyage, sa situation différant de celle du voyageur qui se contente «d'adhérer» à un voyage tout compris.

30 D'après certains auteurs ${ }^{29}$, ce genre de contrats où le voyageur est en quelque sorte le maître de la combinaison est soumis à la LVF. Le Tribunal cantonal vaudois semble confirmer cette opinion, dans un arrêt du 16 février 201630, estimant qu'il « importe [peu] que la combinaison soit imaginée par l'organisateur ou demandée par le voyageur ». En réalité, l'exigence d'une combinaison préalable ne serait qu'un simple indicateur, le critère important étant la confiance que place le voyageur dans son intermédiaire : si celui-ci agit comme un professionnel de la branche en proposant par publicité des prestations touristiques (que le consommateur peut combiner à sa guise) et que le voyageur considère cet intermédiaire comme son partenaire contractuel, alors la LVF s'applique ${ }^{31}$.

31 A ce propos, le droit européen se positionne clairement en faveur du consommateur et d'une acception large de la notion de voyage à forfait. En effet, la nouvelle Directive 2015/2302/UE définit à

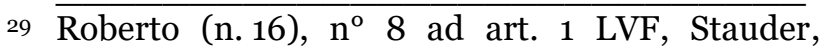
(n. 15), $\mathrm{n}^{\circ} 9$ ad art. 1 LVF, Hangartner Sandro, Das neue Bundesgesetz über Pauschalreisen, thèse de doctorat, Zurich 1997 p. $26 \S 1$, qui parle de «brochure de voyage modulable», Marchand (n. 20), p. 722.

30 Arrêt de la Cour d'appel civile du Tribunal cantonal vaudois du 16 février 2016 in JdT 2016 III 76.

31 ATF 115 II 474 = SJ 1990 216, Stauder (n. 15), $\mathrm{n}^{\circ} 9$ ad art. 1 LVF. 
son art. 3 c. 2 lit. a le forfait comme la combinaison d'au moins deux types différents de services de voyage aux fins du même voyage ou séjour de vacances si ces services sont combinés par un seul professionnel, $y$ compris à la demande du voyageur ou conformément à son choix, avant qu'un contrat unique incluant tous ces services ne soit conclu.

Pour Guyot et Storms ${ }^{32}$, la nouvelle Directive ne fait que reprendre l'arrêt Club Tour33, rendu sous l'empire de l'ancienne Directive 90/314/CEE et dans «lequel [il] avait [été] considéré que les voyages organisés par une agence, à la demande et sur l'initiative du consommateur ou d'un groupe restreint de consommateurs, et conformément à leurs spécifications, relèvent du champ d'application de la notion de voyage à forfait »34. Même si dans le cas d'espèce il s'agissait d'une agence de voyages physique, la doctrine d'en temps estimait que cette considération pouvait valoir également en présence de contrats conclus par Internet35.

33 A notre avis, le droit des voyages à forfait doit s'appliquer dans les cas où la combinaison des prestations émane du voyageur, ce en raison du but de protection du consommateur poursuivi par la LVF et de la nécessité d'interpréter cette loi

32 Guyot Cédric/Storms Fanny, La directive (UE) 2015/2302: en route vers la nouvelle ère des contrats de voyages..., R.E.D.C $\mathrm{n}^{\circ}$ 2016/1, p. 56 $\S 11$.

33 Arrêt de la CJCE C-40o/oo du 30 avril 2002 (Club Tour, Viagens e Turismo c. Alberto Carlos Lobo Gonçalves Garrido9), Rec., 2002, p. 4051.

34 Guyot Cédric/Lanotte Adrien, Le droit européen du tourisme, R.E.D.C n ${ }^{\circ}$ 1/2007-2008, p. 30 \$39.

35 Guyot/Storms (n. 32), p. 56 §11, Führich Ernst, Die neue Pauschalreiserichtlinie, Inhalt und erste Überlegungen zur Umsetzung, NJW 17/2016, p. 1206, voir ég. Wiede (n. 15), p. $168 \S 574$. conformément aux évolutions récentes dans le secteur du tourisme. En effet, avec l'avènement des plateformes en ligne, lesquelles nous l'avons vu laisse une grande liberté de choix aux clients, il n'est guère envisageable de s'en tenir à une interprétation stricte de la loi datant de l'époque des agences de voyages physiques; on ne voit pas en quoi il faudrait péjorer la situation de celui qui peut préciser ses attentes à l'organisateur dont on sait (et cela vaut encore plus dans le cas d'une plateforme) qu'il bénéficie de moyens techniques et personnels 36 importants pour satisfaire le plus de voyageurs possible et ainsi s'offrir la première place du classement.

\section{- Le prix doit être fixé globalement :}

34 La dernière condition posée par la LVF est que le prix du contrat de voyage à forfait soit global. En d'autres termes, il doit être unique, ledit contrat formant alors un tout37. Il n'est cependant pas exclu que certaines prestations soient facturées de manière séparée38.

35 L'on mentionnera encore que la LVF ne s'applique pas aux voyages de durée inférieure à 24 heures ou n'incluant pas de nuitée (art. 1 al. 1 LVF a contrario).

$36 \overline{\text { Nous pensons ici notamment aux multitudes de }}$ fournisseurs qui travaillent, bon gré mal gré, avec les plateformes, à l'image des hôteliers. Sur la question de la concurrence, voir not. la décision de la Commission de la concurrence du 19 octobre 2015 «Online-Buchungsplattformen für Hotels», ainsi que Schiess Daniel/Schaller Olivier, Online-Plattformen - Chancen und $\mathrm{He}$ rausforderungen im Wettbewerbsrecht, in : F. Thouvenin/R. H Weber, Werbung-Online, Center for information, technology, society and law (ITSL), vol. 1, Zurich 2017, p. 109-130.

37 Tercier/Bieri/Carron (n. 25), p. $869 \S 75 n^{\circ} 5862$.

38 Tercier/Bieri/Carron (n. 25), p. $869 \S 75 \mathrm{n}^{\circ} 5862$, Stauder (n. 15), $\mathrm{n}^{\circ} 10$ ad art. 1 LVF, Roberto (n. 16), $\mathrm{n}^{\circ} 10$ ad art. $1 \mathrm{LVF}$. 


\section{b) Les parties au contrat de voyage à forfait}

36

L'art. 2 LVF mentionne trois intervenants au contrat de voyage à forfait, soit l'organisateur, le détaillant et le consommateur. Etant donné que notre étude se focalise sur les plateformes de réservation en ligne, nous nous limiterons aux deux premiers et n'examinerons pas la notion de consommateur au sens du droit des voyages à forfait et nous nous permettons de renvoyer le lecteur à la doctrine correspondante39.

\section{aa) L'organisateur}

La LVF définit l'organisateur comme " toute personne qui, de façon non occasionnelle, organise des voyages à forfait et les offre directement ou par l'intermédiaire d'un détaillant ». Plusieurs éléments ressortent directement ou non de cette définition :

- L'organisateur peut être tant une personne physique qu'une personne morale 40 .

- La tâche de l'organisateur consiste à assembler les différentes prestations touristiques et à les proposer au consommateur41. A cet égard, l'organisateur peut agir directement, mais peut également procéder avec un ou des intermédiaires42 (par ex. des agences de voyages franchisées). Comme le précise Stauder43, «il est sans importance (...) de savoir si

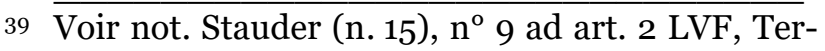
cier/Bieri/Carron (n. 25), p. $871 \S 75 \mathrm{n}^{\circ} 5880$.

40 Stauder (n. 15), $\mathrm{n}^{\circ} 2$ ad art. 2 LVF.

41 Stauder (n. 15), ${ }^{\circ} 2$ ad art. 2 LVF.

42 Tercier/Bieri/Carron (n. 25), p. $870 § 75 \mathrm{n}^{\circ} 5873$, Stauder (n. 15), $\mathrm{n}^{\circ} 2$ ad art. 2 LVF.

43 Stauder (n. 15), ${ }^{\circ} 2$ ad art. 2 LVF. l'organisateur fournit tout ou partie des prestations lui-même ou par son propre personnel, ou si ce sont des prestataires de services indépendants ». Ainsi, une grande latitude est laissée à l'organisateur et il n'est pas rare en pratique que ce dernier s'arme de plusieurs partenaires plus spécialisés que lui.

- L'organisateur doit exercer son activité de façon non occasionnelle. Certains auteurs estiment que l'organisateur doit être un professionnel44, alors que d'autres sont plutôt d'avis qu'il faut exclure du champ d'application de la LVF un organisateur qui offrirait un voyage de temps en temps pour le dire ainsi45. Wiede ${ }^{46}$ propose une analyse intéressante de la doctrine correspondante, et nous nous permettons d'y renvoyer le lecteur.

38 La Directive 2015/2302/UE considère l'organisateur comme un professionnel47, soit une personne exerçant une "activité commerciale, industrielle, artisanale ou libérale »48. Nous estimons que ces termes font référence de près ou de loin à un but lucratif, et que de ce fait la nouvelle règlementation est plus stricte, car elle peut exclure une personne qui organiserait régulièrement des voyages mais sans visée lucrative. Certains auteurs 49 avaient toutefois considéré, déjà sous

44 Tercier/Bieri/Carron (n. 25), p. $870 § 75 n^{\circ} 5873$.

45 Stauder (n. 15), $\mathrm{n}^{\circ} 3$ ad art. 2 LVF, Hangartner (n. 29), p. $25 \S 2$.

46 Wiede (n. 15), pp. 194s.

47 Cf. Art. $3 \S 8$.

48 Cf. Art. $3 \S 7$.

49 Grabitz Eberhard/ Hilf Meinhard, Das Recht der Europäischen Union, Kommentar zur Richtlinie 90/314/EWG, 40ème éd., Munich 2009 art. 2 $\S 12$. 
l'empire de l'ancienne Directive 90/314/CEE, que les organisateurs devaient se soumettre à la réglementation sur les voyages à forfait même s'ils n'agissaient pas dans un cadre commercial.

\section{bb)Le détaillant}

39 Le détaillant est la personne qui offre le voyage à forfait mis sur pied par l'organisateur (art. 2 al. 2 de la LVF) et qui peut être tant une personne physique qu'une personne morale50. Le détaillant ne se charge pas de préparer le voyage, ce rôle incombant à l'organisateur ${ }^{1}$; il a pour fonction principale de proposer le voyage à forfait au voyageur 52 . Il peut s'agir d'une agence de voyages, qui agit pour ses clients, mais également un indépendant spécialiste dans le domaine du tourisme. Nous relevons que la LVF ne fait pas mention de critères tels que «professionnel » ou «non occasionnel » pour qualifier le détaillant. Ainsi, le législateur semble vouloir couvrir le plus de cas possible si bien que l'on ne peut pas exclure d'emblée qu'une personne qui propose gratuitement d'intervenir entre le voyageur et l'organisateur ne soit un détaillant !

Comment distinguer l'organisateur du détaillant ? La LVF traite à plusieurs reprises, en créant la confusion, du «détaillant partie au contrat » (cf. art. 14 al. 1, 17 al. 2). Cette catégorie spéciale recouvre le détaillant qui, aux yeux du consommateur et selon le principe de la confiance, se comporte comme un organisateur (ou à tout le moins, créer

Stauder (n. 15), $\mathrm{n}^{\circ} 5$ ad art. 2 LVF.

Tercier/Bieri/Carron (n. 25), p. $870 \$ 75 \mathrm{n}^{\circ} 5874$.

52 Hangartner (n. 29), p. 26 §2, Roberto (n. 16), $n^{\circ} 8$ ad art. 2 LVF. l'apparence d'être un organisateur)53. Ainsi, cette partie au contrat n'est en réalité pas un détaillant au sens de l'art. 2 LVF, mais un véritable organisateur qu'il convient de traiter comme tel54. Wiede55, auquel nous nous joignons, est d'avis qu'une reformulation de la loi est souhaitable sur ce point.

\section{L'applicabilité de la LVF aux plate- formes de réservation en ligne}

41 Comme nous venons de le voir dans le chapitre précédent, deux critères doivent être remplis pour que la LVF s'applique :

- Un critère matériel, soit un voyage à forfait ;

- Un critère personnel, soit un consommateur, un organisateur et/ou un détaillant.

42 Parmi les plateformes examinées en amont, deux d'entre elles nous semblent remplir ces critères et partant être soumises à la LVF.

\section{a) Là où la LVF est applicable}

43 Nous pensons d'abord à ebookers.ch. Comme relevé précédemment, ses CG prévoient que lorsqu'il y a combinaison d'un vol et d'un hôtel, la LVF s'applique. Aussi, ebookers.ch agit comme une véritable organisatrice professionnelle $\mathrm{du}$ voyage à forfait qu'elle met sur pied et propose ensuite à ses clients. Le fait que ceux-ci puissent choisir différentes modalités, en spécifiant des critères précis, et qu'ebookers.ch compose ensuite le

$53 \overline{\text { Stauder (n. 15), } \mathrm{n}^{\circ} 4 \text { ad art. } 2 \text { LVF, Marchand }}$ (n. 20), p. 723 .

54 Stauder (n. 15), $\mathrm{n}^{\circ} 7$ ad art. 2 LVF.

55 Wiede (n. 15), p. $198 \S 677$. 
voyage ne s'oppose pas à la définition du voyage à forfait comme nous l'avons vu. Relevons encore que si ebookers.ch confie la « vente» de certains de ses voyages à forfait à des tiers, comme une plateforme spécialisée, il ne serait pas exclu d'y voir un détaillant dont les relations avec l'organisateur peuvent être appréhendées de différentes manières 56 .

44 Ebookers.ch ne se contente toutefois pas d'agir en qualité d'organisatrice de voyage à forfait, mais propose séparément des services d'hébergement et de transport en mettant en lien le client avec le fournisseur. Comme la condition de la combinaison des prestations fait défaut, la LVF ne s'appliquera à notre sens pas ; les CG d'ebookers.ch57 prévoient toutefois que le droit suisse est applicable. L'on peut considérer qu'ebookers.ch agit en qualité d'intermédiaire entre un fournisseur, qu'elle représente $5^{8}$, et le client, avec lequel elle conclut un contrat d'intermédiaire de voyage 59 . Bien que l'on distingue traditionnellement le voyage à forfait des prestations individuelles, Wiede ${ }^{60}$ estime toutefois que des liens peuvent exister entre ces deux catégories ; cela est d'autant plus intéressant

$56 \overline{\text { Ainsi, un contrat d'agence (art. } 418 \text { et ss CO) ou }}$ un contrat de courtage (art. 412 et ss CO), cf. not. Stauder (n. 15), $\mathrm{n}^{\circ} 5$ ad art. 2 LVF, Hangartner (n. 30), p. $25 \S 5$, Wiede (n. 15), p. $196 \$ 671$.

57 Voir art. 9 « Loi applicable ».

58 Il n'est ainsi pas exclu d'appliquer les art. 32 et ss CO. La qualification du contrat passé entre ebookers.ch et le fournisseur dépendra essentiellement de la volonté des parties ; l'on peut y voir un contrat d'agence (art. 418a et ss $\mathrm{CO}$ ) ou à tout le moins un contrat de mandat (art. 394 et ss CO).

59 A ce propos, nous nous permettons de renvoyer le lecteur à la contribution de Gauch Peter, Der Werkvertrag, Zurich et al. 2011, p. $140 \mathrm{n}^{\circ} 345$, lequel explique que l'intermédiaire a pour fonction principale de réserver des prestations de voyage pour son client auprès de différents prestataires.

6o Wiede (n. 15), p. $427 \S 1368$. que les portails en ligne proposent directement ou indirectement (ainsi, lorsque le client est redirigé sur un autre site Internet par une fenêtre pop-up) ce genre de prestations touristiques. Nous verrons que le droit européen appréhende, peutêtre de manière incomplète, cette constellation.

45 Concernant ensuite les prestations d'hotelpan.ch, les CG de celle-ci ne mentionnent pas clairement l'application de la LVF61 aux rapports de droit ainsi créés, bien que les termes utilisés dans les CG soient empruntés au droit des voyages à forfait. A notre avis, hotelplan.ch organise des voyages à forfait qu'elle propose à ses clients ; aussi, les critères matériels et personnels d'applicabilité de la LVF nous paraissent remplis. Il est toutefois intéressant de mentionner que le chiffre 4.3.2 des CG d'hotelplan.ch prévoit que «lors de voyages forfaitaires assurés par des voyagistes tiers, MTCH agit en tant qu'intermédiaire ». A notre avis, le raisonnement exposé à propos d'ebookers.ch et du contrat d'intermédiaire de voyage s'applique mutatis mutandis dans cette constellation.

46 Ainsi, le client suisse d'ebookers.ch ou d'hotelplan.ch dispose de différents outils juridiques très développés en matière de voyage à forfait tels que des devoirs d'information accrus (art. 4 et 5 LVF), une protection en cas de modification essentielle du contrat (art. 10 LVF), une protection en cas d'annulation (art. 11 LVF), la responsabilité (art. 14 et Ss LVF), le droit de céder le voyage à un tiers (art. 17 LVF). Toutefois, en matière de presta-

$61 \overline{\text { L'art. } 13 \text { des CG prévoit que « les rapports entre }}$ vous et MTCH sont exclusivement régis par le droit suisse ». 
tion unique de voyage au sens strict, soit lorsqu'aucun lien ne peut être fait avec un voyage à forfait, les droits du voyageur sont plus lacunaires, puisqu'il fait face à deux relations juridiques distinctes :

- Vis-à-vis de la plateforme: nous avons vu qu'il s'agit d'un contrat d'intermédiaire de voyage (cf. supra) soumis aux règles du mandat ${ }^{62}$. Par ce contrat, la plateforme se charge de négocier auprès de différents fournisseurs les prestations qu'elle proposera ensuite à ses clients; en somme, elle constitue un "pont» entre le voyageur et le prestataire.

- Vis-à-vis du prestataire de voyage : le contrat conclu peut prendre différentes formes, comme le contrat de transport de personne soumis aux règles du mandat 63 ou le contrat d'hébergement ${ }^{64}$.

47 A notre avis, cette dualité ne facilite pas la tâche du consommateur lorsque celuici veut faire valoir ses droits ; en effet, il sera parfois difficile de désigner qui est le responsable d'une mauvaise exécution. De plus, et cela vaut aussi dans un contexte plus général, le consommateur peut se sentir bien démuni tant face à une plateforme aux moyens juridiques et financiers importants que vis-à-vis d'un fournisseur bien souvent situé à l'étranger.

62 Arrêt du Tribunal fédéral 4C.125/2004 du 29 juin 2004.

${ }_{63}$ Tercier/Bieri/Carron (n. 25), p. $843 \$ 74 \mathrm{n}^{\circ} 5682$.

64 ATF 120 II 252, Engel Pierre, Le point sur la partie générale du droit des obligations, Entwicklungen im Obligationenrecht, Allgemeiner Teil, RSJ 91/1995 p. 155.

\section{b) Là où la LVF n'est pas applicable}

48 Qu'en est-il des deux autres plateformes que sont booking.com et trivago.ch?

49 Un obstacle majeur surgit lorsqu'il s'agit de leur appliquer la LVF ; en effet, nous avons vu que tant booking.com que trivago.ch ne proposent pas la combinaison de prestations touristiques, mais une seule prestation (le vol ou l'hôtel). Or, l'art.1 LVF exige la combinaison du transport et de l'hébergement, ou de l'une de ces prestations avec d'autres services touristiques non accessoires. Dès lors, cette exigence rigide du texte légal semble clairement s'opposer à l'application de la LVF à ces deux plateformes. Aussi, nous allons successivement examiner les solutions offertes par le droit européen (i), lesquelles pourraient inspirer le législateur suisse en cas de réforme du droit des voyages à forfait, puis nous analyserons quelques questions liées au droit international privé (ii).

\section{aa) En droit européen}

50 La situation serait ici différente et ce pour au moins deux raisons. Précisons d'emblée que l'application du droit européen au sein même des Etats membres est quelque peu précipitée, étant donné que ceux-ci ont encore jusqu'au $1^{\mathrm{er}}$ janvier 2018 pour transposer la nouvelle Directive dans leur droit interne. Toutefois, les CG des deux plateformes contiennent des élections de droit en faveur respectivement du droit hollandais (booking.com) et du droit allemand (trivago.ch), raison pour laquelle nous avons décidé d'examiner la nouvelle réglementation européenne. 
La définition du voyage à forfait: L'art. $3 \quad \S 2$ let.b de la Directive 2015/2302/UE définit le voyage à forfait comme la combinaison d'au moins deux types différents de services de voyage aux fins du même voyage ou séjour de vacances, si indépendamment de l'éventuelle conclusion de contrats séparés avec des prestataires de services de voyage individuels, ces services sont achetés auprès de professionnels distincts grâce à des procédures de réservation en ligne liées. Il faut, dans ce cas, que le nom du voyageur, les modalités de paiement et l'adresse électronique soient transmis par le professionnel avec lequel le premier contrat a été conclu à un ou plusieurs autres professionnels et que le contrat avec ce ou ces derniers soit conclu au plus tard 24 heures après la confirmation de la réservation du premier service de voyage.

Il s'agit par ce biais d'éviter que les organisateurs de voyage n'éludent la Directive 2015/2302/UE en ne combinant pas des prestations, mais en proposant des contrats séparés. Führich 65 estime que l'on vise ici les "contrats de transmission" par lesquels un premier professionnel, comme la compagnie aérienne, transmet les données du client à un autre professionnel, typiquement l'hôtelier, auprès duquel un contrat d'hébergement est conclu. L'art. $7 \& 3$ de la Directive 2015/2302/UE règle les relations entre ces différents intervenants, de manière à créer « fictivement » un forfait ${ }^{66}$. Ainsi, il ne serait pas exclu d'appliquer la régle-

65 Führich (n. 35), p. 1206, voir ég. Ineichen Reto, Umsetzung der neuen EU-PauschalreiseRichtlinie in der Schweiz - Chance oder No Go?, RRa 1/2017 p. 3, qui parle de «Click-TroughBuchung».

66 Führich (n. 35), p. 1206. mentation sur les voyages à forfait (européenne mais transposée en droit interne) à booking.com ou trivago.ch si elles transmettent les coordonnées du client à une autre plateforme.

53 Les prestations de voyage liées (ciaprès : PVL) : La Directive 2015/2302/ UE instaure une nouvelle notion, «les prestations de voyage liées ", soit au moins deux types différents de services de voyage achetés aux fins du même voyage ou séjour de vacances, ne constituant pas un forfait, mais entraînant la conclusion de contrats séparés avec des prestataires de services de voyage individuels, si un professionnel facilite :

- à l'occasion d'une seule visite à son point de vente ou d'une seule prise de contact avec celui-ci, le choix séparé et le paiement séparé de chaque service de voyage par les voyageurs ; ou

- d'une manière ciblée, l'achat d'au moins un service de voyage supplémentaire auprès d'un autre professionnel lorsque le contrat avec cet autre professionnel est conclu au plus tard 24 heures après la confirmation de la réservation du premier service de voyage.

54 D'après Guyot et Storms ${ }^{67}$, l'idée du législateur européen est de permettre au voyageur de choisir et modeler son voyage comme il le souhaite et ainsi lui conférer une certaine liberté, tout en lui garantissant une protection. Comment distinguer le voyage à forfait des PVL ?

67 Guyot/Storms (n. 32), p. $57 \S 13$. 
L'Institut National de la Consommation français ${ }^{68}$ donne l'exemple suivant :

1. A réserve un vol sur un site Internet. Il réserve également une nuit d'hôtel sur un site différent, auprès duquel il a été « redirigé » pas des procédés de réservation en ligne liés. Dans ce cas, A conclut un contrat de voyage à forfait (pour autant qu'il y ait eu un transfert de ses données dans les 24 heures après la réservation du vol auprès la compagnie aérienne).

2. Même cas de figure, mais le site de réservation, par exemple booking.com, n'a pas transmis ces données à l'autre plateforme de réservation en ligne vers laquelle $\mathrm{A}$ a été redirigé ; dans ce cas, A a conclu séparément des contrats avec différents prestataires de services, lesquels tombent sous la notion de PVL.

Filliâtre ${ }^{69}$ résume en ces termes ce qu'est une PVL : "c'est un genre de forfait déguisé qui combine plusieurs types de prestations délivrées par des intermédiaires séparés ". Le client $\mathrm{A}$, dans notre second exemple, crée son voyage en réservant d'abord un vol, puis il clique sur un site lui proposant une chambre d'hôtel (étonnamment dans la ville choisie et aux bonnes dates !) MAIS il n'y a aucun lien entre ces deux professionnels, si ce n'est une transmission de données. Mais qu'est-ce qui change concrètement? Alors que la nouvelle Directive consacre tout un pan d'obligations pour

$68 \overline{\text { L'Institut National de la Consommation, Voyage }}$ à forfait : la nouvelle directive publiée !, 12 janvier 2016.

69 Filliâtre Pascale, Nouvelle directive sur les voyages à forfait : ce qu'il faut savoir, l'echo touristique du 7 octobre 2016. les professionnels de la branche voyage à forfait (informations précontractuelles, droit de résiliation, etc.), seuls deux droits sont conférés aux voyageurs qui sont liés par un contrat de PVL :

- Une protection contre l'insolvabilité (art. $\quad 19 \quad \S \quad 1$ de la Directive 2015/2302/UE) : la protection est sensiblement la même que celle en matière de voyage à forfait ;

- Des obligations d'informations (art. $19 \S 2$ ) : le voyageur doit être renseigné négativement ${ }^{70}$ sur le fait qu'il ne bénéficiera pas des droits applicables aux voyages à forfait. Si le professionnel omet de se conformer à cette exigence, le voyageur bénéficiera des droits prévus aux art. 9 et 12 de la Directive, soit les droits de cession et de résiliation.

56 Quel bilan tirer de cette nouvelle notion? Selon certains auteurs ${ }^{71}$, auxquels nous adhérons, prévoir des obligations moindres pour les professionnels proposant des PVL alors que les professionnels des voyages à forfait croulent sous une réglementation contraignante, c'est ouvrir la porte à des changements de plan économique: les professionnels de la branche du tourisme seraient tentés de se tourner exclusivement vers les PVL, en abandonnant le voyage à forfait classique. Et c'est le consommateur qui en ferait les frais, alors que la nouvelle réglementation vise justement... à le protéger!

$70 \overline{\text { Poillot Elise, Droit de la consommation : actuali }}$ tés législatives, Journal de droit européen 2016 p. $281 \S 3$.

71 Guyot/Storms (n. 32), p. 58 §16, Führich (n. 35), p. 1207. 


\section{bb)En droit international privé}

57 Comme booking.com et trivago.ch ont leur siège social respectivement au PaysBas et en Allemagne ${ }^{72}$ et que notre voyageur est par hypothèse domicilié en Suisse, la réflexion peut s'élargir pour aborder des questions de droit international privé. En particulier, deux points devraient être analysés :

- La question de la compétence des tribunaux : puisque les Etats concernés sont parties à la Convention de Lugano (ci-après CL)73, les art. 15 à $17 \mathrm{CL}$ pourraient entrer en ligne de compte, pour autant que notre consommateur conclut un contrat pour un usage pouvant être considéré comme étranger à son activité professionnelle, et ce, peu importe le genre de contrat conclu, ce qui compte étant «la raison (...) pour laquelle l'accord est conclu »74. Ainsi, un contrat de mandat par lequel la plateforme s'engage à fournir un portail permettant la comparaison et la réservation d'un hôtel pourrait tomber dans le champ de l'art. 15 CL. En outre, si le professionnel dirige son activité vers le pays dans lequel réside le consommateur (ce qui n'est pas sans poser des difficultés en présence du commerce électronique) 75 , alors le voyageur-consommateur

72 Trivago.ch n'est toutefois pas très limpide à ce propos, « Legal Information_».

73 Convention concernant la compétence judiciaire, la reconnaissance et l'exécution des décisions en matière civile et commerciale (Convention de Lugano, RS 0.275.12).

74 Bonomi A., in : A. Bucher (édit.), Commentaire romand, Loi sur le droit international privé, Convention de Lugano, Bâle 2011, $\mathrm{n}^{\circ} 19$ ad art. 15 CL.

75 Bonomi (n. 74), $\mathrm{n}^{\circ} 42$ ad art. 15 CL, Fornage (n. 20), p. $252 \S 1081$. pourra attenter action auprès des tribunaux de son domicile soit les tribunaux suisses (art. $16 \mathrm{CL}$ ), l'élection de for étant admise de manière restrictive $^{76}$ par l'art. 17 CL.

- La question du droit applicable : la Loi fédérale sur le droit international privé (ci-après LDIP) 77 contient une protection spéciale en faveur des consommateurs, puisque l'art. 120 LDIP prévoit l'application du droit de l'Etat de la résidence habituelle du consommateur (dans notre cas le droit suisse) dans certaines circonstances, l'élection de droit étant par ailleurs prohibée. Il faut que le contrat porte sur une prestation de consommation courante destinée à un usage personnel ou familial du consommateur; or, la loi vise ici «toutes sortes de contrats entre consommateurs et fournisseurs $\gg 78$, peu importe en somme leur contenu. Ainsi, le contrat passé entre la plateforme et le voyageur peut tomber sous le coup de l'art. 120 LDIP.

58 Toutefois, l'on relèvera que le droit de la résidence habituelle du consommateur n'est pas forcément le plus avantageux pour notre voyageur79; en effet, nous avons vu que le droit européen présente déjà des solutions pour appréhender les plateformes de réservation en ligne, ce

76 Bonomi (n. 74), $\mathrm{n}^{\circ} 1$ ad art. 17 CL, M. A Gehri, in : C. Oetiker/T. Weibel (édit.), Basler Kommentar, Lugano-Übereinkommen, 2 ème éd., Bâle 2016, $\mathrm{n}^{\circ} 2$ ad art. 17 CL.

77 Loi fédérale du 18 décembre 1987 sur le droit international privé (RS 291).

78 ATF 121 III 336, Dutoit Bernard, Droit international privé suisse, Commentaire de la loi fédérale du 18 décembre 1987, 5éme éd., Bâle 2016, $\mathrm{n}^{\circ} 4$ ad art. 120.

79 Bonomi (n. 74), n 23 ad art. 120 LDIP. 
qui n'est pas encore le cas du droit suisse $^{80}$.

\section{Conclusion}

59 Ainsi s'achève le voyage juridique parmi les plateformes de réservation en ligne, guidé par la LVF et le droit européen. L'application de la législation suisse à ce nouveau mode de consommation n'est pas aussi aisée que l'on pourrait le penser, et il serait temps que notre législateur prenne le premier vol pour un changement en matière de voyages à forfait : Internet est venu chambouler la conclusion des contrats et implique souvent plusieurs acteurs, dont il est parfois difficile de définir le rôle. En effet, comment appréhender juridiquement des relations multilatérales, alors que les plateformes semblent vouloir s'en laver les mains? Le droit européen a su saisir ces implications, peut-être de manière imparfaite, alors que notre droit est encore une fois à la traîne, le secteur du voyage en général n'intéressant (malheureusement) pas tant les juristes. Reste que ce domaine est en pleine expansion et que les plateformes prennent de plus en plus de place dans nos vies!

Cf. toutefois le Rapport du Conseil fédéral du 11 janvier 2017 sur les principales conditions-cadre pour l'économie numérique, pp. 73-74, lequel estime que les relations entre le client et la plateforme relèvent du contrat d'agence, du courtage ou du mandat, lorsque ladite plateforme considère agir en simple qualité d'intermédiaire, soit lorsqu'elle n'a aucune obligation d'effectuer la prestation caractéristique. 